\title{
Playing the e-Business Game in 3D Virtual Worlds
}

\author{
Helmut Berger \\ E-Commerce \\ Competence Center - EC3 \\ A-1220 Wien, Austria \\ helmut.berger@ec3.at \\ Anton Bogdanovych \\ Faculty of IT, University of \\ Technology Sydney \\ Sydney, NSW, Australia \\ anton@it.uts.edu.au
}

\author{
Michael Dittenbach \\ E-Commerce \\ Competence Center - EC3 \\ A-1220 Wien, Austria \\ michael.dittenbach@ec3.at \\ Simeon Simoff \\ Faculty of IT, University of \\ Technology Sydney \\ Sydney, NSW, Australia \\ simeon@it.uts.edu.au
}

\author{
Dieter Merkl \\ Institut für rechnergestützte \\ Automation, TU Wien \\ A-1040 Wien, Austria \\ dieter.merkl@inso.tuwien.ac.at \\ Carles Sierra \\ Artificial Intelligence \\ Research Institute (IIIA) \\ Barcelona, Catalonia, Spain \\ sierra@iiia.csic.es
}

\begin{abstract}
In this paper we present an integrated, game-like eBusiness environment that follows the role model of Massively Multi-User Online Role-Playing Games (MMORPGs). The interface is realized as a 3D virtual world using affordable game engine technology. Our environment provides a platform for conducting business and it is supposed to be a community facilitator to create and establish a lively and sustainable online community involving both, providers and consumers. It is information-rich and multimedia-based offering transparent access to disparate information sources.
\end{abstract}

\section{Author Keywords}

3D Virtual Worlds, Game Engines, Online Communities, e-Business, Multi-Agent Systems

\section{ACM Classification Keywords}

H5.2. HCI: User Interfaces; H4.3. INFORMATION SYSTEMS APPLICATIONS: Communications Applications

\section{INTRODUCTION}

The design and development of virtual worlds has emerged as a phenomenon shaped by the home computer user rather than by research and development activities at universities. Bricken (1991) identified the shift from a passive user role to participation in the actual design, the move from interface towards inclusion, i.e. involving participants in the design process within the environment, and the change from visual to multimodal interaction. The development and research in distributed gaming environments as well as in computer-mediated collaborative design identified the need of dynamic generation of virtual worlds from design specifications. For example, Smith et al. (2003) changed static 3D virtual worlds into adaptable worlds by incorporating software agents as the basis for representing the world's elements.

Individuals are the product of a particularly mobile and

OZCHI 2006, November 20-24, 2006, Sydney, Australia. Copyright the author(s) and CHISIG

Additional copies are available at the ACM Digital Library (http://portal.acm.org/dl.cfm) or ordered from the CHISIG secretary (secretary@ chisig.org)

OZCHI 2006 Proceedings ISBN: 1-59593-545-2 entrepreneurial society. As a result, individuals are socially constituted and socially situated in everyday business activities. Preece \& Maloney-Krichmar (2003) criticize that the satisfaction of social needs, despite its great importance, is widely neglected in contemporary interactive systems. A truly feasible e-Business system that supports business activities can hardly be obtained without taking care of the social issues behind these activities (Wyckoff \& Colecchia, 1999). Some operators of e-Business systems even believe that online communities supporting social interactions serve the same purpose as the "sweet smell of baking cakes" does in a pastry shop. Both evoke images of comfort, warmth, happiness and probably even trust. Most system analysts, however, perceive such systems from a purely technical viewpoint neither bearing in mind the social norms that companies and consumers comply with nor acknowledging the importance of human consultancy in a decision making process. 3D virtual worlds address the satisfaction of users' social needs and are complemented with a realistic experience. Virtual worlds support the way humans act and communicate in real life to a certain extent and offer an environment to meet people. Such interfaces go beyond the form-based approaches dominating the Internet and graphically represent the user in terms of an avatar (Damer, 1998). Users are literally in the Internet rather than on it. 3D virtual worlds implicitly address the issue of social interactions since location awareness, presence, as well as direct communication are intrinsic elements.

In this paper we present an integrated, game-like eBusiness environment that is based on a multi-agent system. The role model for our environment is the concept of MMORPGs. Millions of users interact, collaborate, socialize and form relationships with each other through avatars in such online environments (Castronova, 2005). We address the aspect of social interaction by providing instruments to interact and to exchange experiences with other customers that go beyond the possibilities of conventional text-based chat rooms. So, the environment acts as the catalyst for the creation of a sustainable online community. Additionally, the environment provides a platform for conducting business, i.e. buying and selling of goods. Our environment enables seamless access to the information richness of the Internet within a 3D virtual world. A 3D 
game engine is used to provide an affordable solution for the creation of the $3 D$ virtual world. Similar to the 3D virtual museum described in (Lepouras \& Vassilakis, 2004), our environment offers an immersive and realistic experience accessible from the user's desktop computer.

\section{THE 3D E-BUSINESS ENVIRONMENT}

\section{Design Considerations}

Multi-agent systems have proven to be a suitable paradigm for modelling environments that are composed of many autonomous individuals. In order to develop complex multi-agent systems, sophisticated methodologies supporting the entire development life cycle including design, analysis and deployment are needed (Jennings et al., 1998). Electronic Institutions (Sierra, 2004), a prominent representative of such methodologies, are used to model electronic negotiations in our e-Business environment. Electronic negotiations promise a higher degree of efficiency and effectiveness, and most importantly, a higher quality and faster emergence of agreements. The goal is to enable the trade of complex and valuable goods which entails a negotiation process consisting of a number of communicative exchanges (Fatima et al., 2005).

3D Electronic Institutions combine the two paradigms of Electronic Institutions and 3D virtual worlds while retaining the features and advantages of both. 3D Electronic Institutions are environments that enable humans to participate in a heterogeneous society of individuals visualized in a $3 \mathrm{D}$ virtual world. The essence is to transcend the agent-centered view of Electronic Institutions, take a human-centered perspective and concentrate on the complex relationship between humans and agents in the amalgamation of the two paradigms (Bogdanovych et al., 2005). We consider two types of participants, namely humans and software agents, building a heterogeneous society. A software agent is either controlled by a human or acts autonomously. In the first case, the human is the principal of her software agent. The couple principal/software agent is represented as an avatar in the 3D virtual world. Human and software agent cooperate in order to collaboratively achieve a particular goal. Cooperation ranges from delegating simple tasks such as information gathering to complex ones as, for instance, participating in electronic negotiations. In the second case, software agents render the environment information-rich. They access external, disparate information sources, aggregate collected information, and present it as an integral part of the environment.

Our environment is designed to emulate the way humans operate and interact in the real world. 3D virtual worlds aim at combining the use of space with an immersive experience in order to construct a usable virtual representation of a particular application domain. Space and objects in space are used to model different impressions. Proximity of objects indicates that they belong to the same group or are of a similar type. A particular role of a participant in the virtual world is represented by means of a specific outfit. To this end, an avatar dress code that allows the perception of visual cues associated to the roles of participants is introduced. For example, the visualization of a retailer is constrained such that the avatar needs to be dressed in a specific workwear. Since an avatar can either be controlled by a human or a software agent, this aspect has to be made obvious by means of explicit visual cues. In case of autonomously acting software agents, the visual representation depends on their task. In other words, a human-like representation is not necessarily appropriate. The obvious appearance of a software agent responsible for streaming a video clip featuring a particular product is a video wall.

Virtual worlds visualized in $3 \mathrm{D}$ are environments where people meet. Such environments provide a consistent interface metaphor that facilitates awareness of other participants. Communication and interaction between participants are central in these environments. Being aware of other users constitutes an implicit and integral feature of this user interface metaphor and offers communication possibilities at any time detached from any physical place. Text-based communication is realized by means of a chat facility offering a synchronous mode of interaction. However, in a sustainable multi-user environment it is also necessary to provide mechanisms for asynchronous communication. This is realized by Instant Messaging, which integrates e-Mail, bulletin boards as well as information digesting services.

Recent reports indicate that lurkers make up over $90 \%$ of online groups (Nonnecke \& Preece, 2000). Invisibility of participants, as it is possible in most web-based forums, is not an issue in our environment. However, people may still stand around inactively not talking to anybody. In order to lure out these lurkers and encourage active participation in the online community we introduce several counteracting methods. Firstly, a special tutorial area for newcomers and newbies, the technically challenged or the shy, helps to get to know the environment and to establish first social contacts. Secondly, the level of active participation within the community is monitored and, in case of long lasting inactivity, special animators are provided by the environment. The task of these animators is to engage passive members in conversations, ask if they need assistance, provide pointers and hints regarding their interests, and so forth. Thirdly, each participant has the possibility to explicitly communicate her particular quest by attaching a visual cue to her avatar. The concept of quest is borrowed from the area of MMORPGs where a participant may indicate, say, "Looking for work" or "Looking for friend" by means of a textual label hovering above her avatar's head. In our context, this might range from simple statements such as "Looking for information" to specific ones such as "Need shared accommodation in Oslo".

Godwin (1994) argues that online communities need to provide durable records of the history of the community members. This encourages the development of reputations, which can be a vital source of social information and control. The participant's reputation is influenced by the amount of helpful advices provided. 
This alters the look of the avatar to make it obvious to others that this person is a valued participant with expertise in, say, the Asian stock market.

\section{System Architecture}

Basically, the architecture follows the three-layered framework of 3D Electronic Institutions. For the multiagent system layer Electronic Institutions are employed. This particular layer hosts the runtime environment AMELI for arbitrary Electronic Institutions. These institutions are specified with ISLANDER (Esteva et al., 2002), a UML-like editor that verifies the institution with respect to integrity, protocol correctness, and norm correctness. AMELI loads an institution specification and mediates the interaction of software agents while enforcing institutional rules and norms. To execute an Electronic Institution, AMELI is launched up-front and software agents join the institution by connecting to the runtime environment. An Electronic Institution is in a sense a natural extension of the socio-economic concept of institutions as regulatory systems shaping human interactions (North, 1991).

The middleware causally connects the multi-agent system to the visualization layer. As Maes \& Nardi (1988) point out, a system is "causally connected" to its representation when the following facts apply. Firstly, whenever the representation of a system is changed, the system itself has to change as well. Secondly, whenever the system evolves, its representation has to be modified in order to maintain a consistent relationship. So, the causal connection materializes in two directions. Messages sent by the software agent in the multi-agent system have immediate impact on the 3D representation. Actions performed by the human in the $3 \mathrm{D}$ virtual world are translated to messages sent by the software agent.

The third layer contains the user interface that is realized using a 3D game engine. We have conducted an evaluation of several commercial and non-commercial 3D game engines whereof the Torque Game Engine by GarageGames turned out to be the most adequate. This particular game engine provides a comprehensive set of design and development tools including a World Editor, a GUI Editor and a Terrain Editor, which perfectly assist during the creation of arbitrary games executable on all major operating systems. Moreover, it offers multi-player network code, seamless indoor/outdoor rendering engines, state of the art skeletal animation, drag and drop GUI creation, and a C-like scripting language. In addition to that and unlike most commercial game engines the source code of the engine is distributed as part of the low cost royalty-free licensing policy, which facilitates the creation of the $3 \mathrm{D}$ environment.

\section{An e-Tourism Showcase}

Tourism products cannot be observed or manipulated through direct experience prior to purchase - they are confidence goods. An a priori assessment of product quality is virtually impossible and customers need to rely on indirect or virtual experience when making their decisions (Liu, 2005). Consequently, appealing presentations of products, e.g. travel destinations, have always been an important success factor in tourism. In a nutshell, we consider sophisticated visualization of tourism products, the consulting role of travel agents, the social interaction and information exchange between travelers, as well as the information richness of the Internet as being the key features for successful eBusiness in tourism.

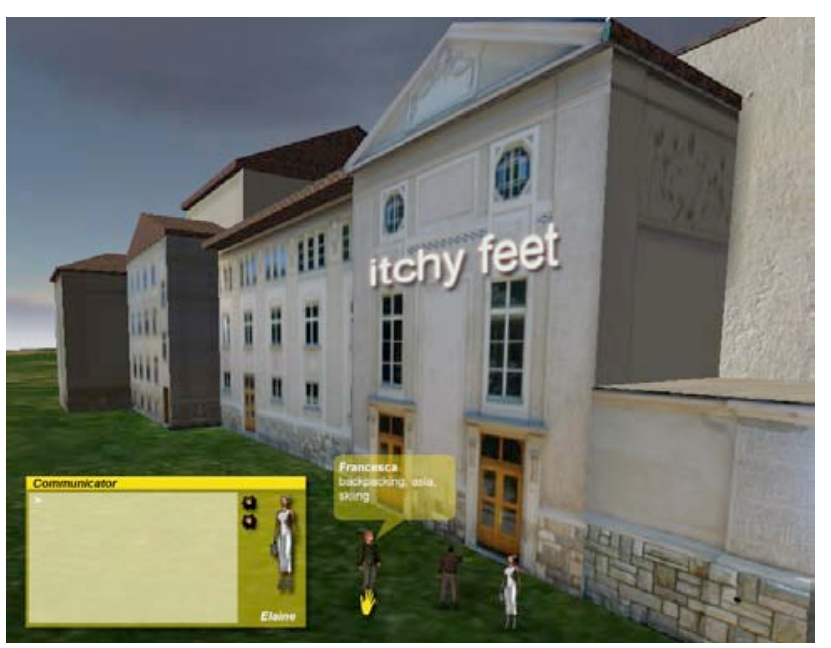

Figure 1. The entrance to the premises of "itchy feet".

Thus, we are currently developing a system that embraces all of these diverse issues and showcase first visual impressions in Figures 1 and 2 . The e-Tourism environment "itchy feet" is visualized in terms of a 3D representation of a building surrounded by a grassy area. As soon as a participant enters the e-Tourism environment she is impersonated as an avatar and positioned near the premises of "itchy feet". In this particular case, Figure 1 depicts the view of Elaine. The appearance of Elaine's avatar is guided by the preferences kept in her profile. Moreover, the profile stores information on her travel likings, special interests and maintains a history of interactions she made during previous sessions. Elaine interacts with the environment via mouse and keyboard. The mouse is used to change the viewpoint and to trigger events such as opening doors, selecting other participants, etc. If the mouse is pointed towards an avatar, the individual's name as well as her interests are displayed in a transparent bubble. In this case, Elaine points her mouse on Francesca and reveals that she is interested in backpacking, skiing and traveling Asia. The lower left corner of the interface features the interaction module, i.e. the Communicator. This module allows to chat with other participants including software agents, to obtain messages regarding the status of the environment, e.g. number of participants, to receive news from the maintainer of the e-Tourism environment, and to send and receive mail. Additionally, the Communicator is used to change the appearance of the avatar. This is accomplished by clicking on the little figure to the right of the Communicator window which opens the repository of available avatars, gestures and accessories such as clothes or bags. The premises of "itchy feet" is accessed via the main entrance located beneath the "itchy feet" sign, cf. Figure 1. The interior contains different areas including a room for conducting business such as booking 
trips or auctioning, an area for information gathering and information exchange, a section devoted to gaming as well as a community area, the Travelers' Lounge as shown in Figure 2. This area enables participants to engage in conversations, talk about experiences they made during their travel, recommend or dissuade particular tourism destinations or just enjoy a relaxed gettogether with other travel addicts. In this particular case, Figure 2 depicts the view of Sebastien, impersonated as an avatar, on the Travelers' Lounge. Besides Sebastien, a number of other visitors are present, some engaged in a conversation or about to join, others just waiting and observing the scene. The two video walls in the rear of the room are visual representations of software agents that deliver information about particular travel destinations based on the requests of participants standing in front of the video walls.

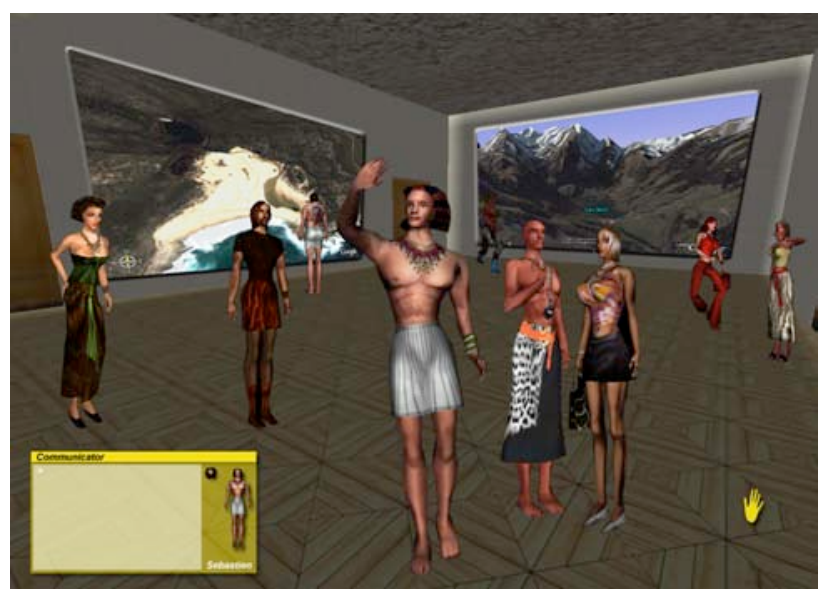

Figure 2. The Travelers' Lounge.

\section{CONCLUSIONS}

Considering the current success of Massively Multi-User Online Role-Playing Games on the one hand, and the growing market share of products being bought online on the other hand, the combination of entertainment and business has the potential of creating enormous synergies. To this end we have presented an integrated, game-like eBusiness application where participants, either humans or software agents, are embodied as avatars in a multi-agent based 3D virtual world. Its goal is to support the complex interaction patterns between the members of this heterogeneous society in an e-Business setting. This environment provides visualization of and interaction with products, supports business activities and provides the grounds for a lively online community that fosters social interaction. The innovative approach of marrying affordable gaming technology with electronic business elevates interaction with serious e-Business applications to a more social, joyful and playful experience.

\section{REFERENCES}

Bogdanovych, A., Berger, H., Simoff, S. \& Sierra, C. (2005), Narrowing the gap between humans and agents in e-commerce: 3D Electronic Institutions, in 'Proc. of the 6th Int'l Conf. on Electronic Commerce and Web Technologies (EC-Web'05)', Copenhagen, Denmark, pp. 128-137.
Bricken, M. (1991), 'Virtual worlds: No interface to design.', Cyberspace: First steps pp. 363-382.

Castronova, E. (2005), Synthetic Worlds: The business and culture of online games, University of Chicago Press, Chicago, IL.

Damer, B. (1998), Avatars: Exploring and Building Virtual Worlds on the Internet, Peachpit Press.

Esteva, M., de la Cruz, D. \& Sierra, C. (2002), ISLANDER: An electronic institutions editor, in 'Proc. of the 1st Int'l Conf. on Autonomous Agents and Multiagent Systems', Bologna, Italy, pp. 1045-1052.

Fatima, S., Wooldridge, M. \& Jennings, N. (2005), 'A comparative study of game theoretic and evolutionary models of bargaining for software agents', Artificial Intelligence Review 23, 185-203.

Godwin, M. (1994), 'Nine principles for making virtual communities work', Wired 2.06, 72-73.

Jennings, N., Sycara, K. \& Wooldridge, M. (1998), 'A roadmap of agent research and development', Autonomous Agents and Multiagent Systems 1(1), 738.

Lepouras, G. \& Vassilakis, C. (2004), 'Virtual museums for all: Employing game technology for edutainment', Virtual Reality 8(2), 96-106.

Liu, S. (2005), A theoretic discussion of tourism ecommerce, in 'Proc. of the 7th Int'l Conf. on Electronic Commerce (ICEC'05)', ACM Press, Xi'an, China, pp. $1-5$.

Maes, P. \& Nardi, D. (1988), Meta-Level Architectures and Reflection, Elsevier Science Inc., New York, NY.

Nonnecke, B. \& Preece, J. (2000), Lurker demographics: counting the silent, in 'Proc. of the SIGCHI Conf. on Human Factors in Computing Systems (CHI'00)', ACM Press, The Hague, The Netherlands, pp. 73-80.

North, D. (1991), Institutions, Institutional Change and Economic Performance, Cambridge University Press, Cambridge, UK.

Preece, J. \& Maloney-Krichmar, D. (2003), Online communities: Focusing on sociability and usability, in J. Jacko \& A. Sears, eds, 'The Human-Computer Interaction Handbook', Lawrence Erlbaum Associates Inc., Mahwah, NJ, pp. 596-620.

Sierra, C. (2004), Electronics Institutions: Methodology of multi-agent systems development., in 'Proc. of the 8th Pacific Rim Int'l Conf. on Artificial Intelligence (PRICAI'04)', Springer Verlag, Auckland, New Zealand, pp. 1-2.

Smith, G., Maher, M. \& Gero, J. (2003), Designing 3d virtual worlds as a society of agents, in 'Proc. of the 10th Int'1 Conf. on Computer Aided Architectural Design Futures', Tainan, Taiwan, pp. 105-114.

Wyckoff, A. \& Colecchia, A. (1999), The Economic and Social Impacts of Electronic Commerce: Preliminary Findings and Research Agenda, Organization for Economic Cooperation and Development. 